\title{
Comparison of Sequential feeding and Continuous feeding on Blood Glucose of critically ill patients: A non-inferiority Randomized Controlled Trial
}

\section{Changjie Ren}

the affiliated hospital of Qingdao university

\section{Bo Yao}

the affiliated hospital of Qingdao university

Miao Tuo

the affiliated hospital of Qingdao university

Hui Lin

the affiliated hospital of Qingdao university

\section{Xiangyu Wan}

the affiliated hospital of Qingdao university

Xufeng Pang ( $\nabla$ qdfyicuhl@163.com )

Qingdao Women and Childrens Hospital https://orcid.org/0000-0001-8761-3595

\section{Research article}

Keywords: intensive care unit; enteral feeding; blood glucose; feeding intolerance

Posted Date: August 25th, 2019

DOI: https://doi.org/10.21203/rs.2.13101/v1

License: () (1) This work is licensed under a Creative Commons Attribution 4.0 International License. Read Full License

Version of Record: A version of this preprint was published at Chinese Medical Journal on July 20th, 2021. See the published version at https://doi.org/10.1097/CM9.0000000000001684. 


\section{Abstract}

Objective: The goal of this study is to compare sequential feeding and continuous feeding on blood glucose of critically ill patients. Methods: A non-inferiority randomized controlled trail was adopted in this study. A total of 62 patients were finally enrolled, who were fed with enteral nutritional suspension through gastric tubes. After achieving $80 \%$ of the nutrition target calories $(25 \mathrm{kcal} / \mathrm{kg} / \mathrm{d})$ through continuous feeding, the patients were then randomly assigned into sequential feeding and continuous feeding group. In sequential feeding group, the feeding/fasting time was reasonably determined, according to the circadian rhythm of the human body in traditional Chinese medicine theory. The total daily dosage of enteral nutritional suspension was equally distributed to the three time periods of 7-9 o 'clock, 11-13 o 'clock and 17-19 o 'clock. Enteral nutritional suspension in each time period was pumped at a uniform rate within two hours by enteral feeding pump. While in continuous feeding group, patients received continuous feeding with constant velocity by enteral feeding pump during the study. Blood glucose values at 5 points (6:00/11:00/15:00/21:00/1:00) were monitored and recorded in 7 consecutive days after randomization. Meanwhile, enteral feeding intolerance was also recorded. Results: No significant demographic or physiologic differences between the sequential feeding and continuous feeding group $(P>0.05)$. The average of glucose in sequential feeding was no inferior to continuous feeding [8.8(7.3-10.3) $\mathrm{mmol} / \mathrm{L}$ vs. 10.7(9.1-12.1) $\mathrm{mmol} / \mathrm{L}, \mathrm{P}$ for non-inferiority $=0.038)$. Hyperglycemia in CF group was more often than SF group (36.1\% vs. $23.0 \%, P \otimes 0.01)$. There was no significant differences in glucose standard deviations and Glucosemax -Glucosemin between two groups (P囚0.05). But the coefficient of glucose variation in sequential feeding group was higher than continuous feeding group [24.5(22.2-27.6) \% vs. 18.9(13.7-25.3) \%, P=0.013). Moreover, no significant difference in the incidence of feeding intolerance during the 7 days were found $(P>0.05)$. Conclusions: In this non-inferiority study, average blood glucose value of critically ill patients with sequential feeding was not higher than continuous feeding. And the feeding intolerance in sequential feeding was similar to continuous feeding.

\section{Introduction}

It is recommend that ICU patients should start enteral feeding as long as gastrointestinal function allows. However, most critically ill patients have physiological dysfunction, poor intestinal tolerance, and are prone to feeding complications. Continuous feeding, due to long duration of infusion, may have benefits in enteral feeding complications. Compared to intermittent feeding, continuous feeding is thought to be better tolerated by critically ill patients, better controlling of blood glucose and lower incidence of feeding complications such as diarrhea, vomiting, and aspiration ${ }^{[1]}$. However, in recent years, according to relevant literature reports, continuous enteral nutrition feeding methods have irrational problems such as biology, protein synthesis, gastric volume and physiological functions, which do not conform to the physiological state of the human body, and violate the biological rhythm of the human body ${ }^{[1-2]}$.

Moreover, with the deepening of research and the increase in the number of included cases, more and more researches have shown that the benefits in enteral feeding complications of continuous feeding was not less than intermittent feeding ${ }^{[3-5]}$. A randomized three-way crossover pilot study of healthy 
volunteers showed no difference in gastric reflux between intermittent and continuous feeding ${ }^{[3]}$. For critically ill patients, continuous feeding and intermittent feeding have no difference in enteral feeding complications such as diarrhea, bloating and vomiting ${ }^{[4]}$. No clinically relevant differences in glycemic variability and insulin use were found between bolus tube feeding and continuous tube feeding ${ }^{[5]}$.

Under normal physiological conditions, the circadian system readies the body for daytime feeding. The rate of gastric emptying and gastrointestinal motility is faster in the daytime than at night, which rates peak in the morning, and the basic gastric acid secretion also has its internal circadian rhythm as well ${ }^{\text {[6- }}$ 8]. Aligning feeding/fasting cycles with clock-regulated metabolic changes optimizes metabolism, and studies of other animals suggest that feeding at inappropriate times disrupts circadian system organization and thereby contributes to adverse metabolic consequences and chronic disease development ${ }^{[9]}$. Now, we firstly proposed a new feeding method, which is called Sequential Feeding. In this sequential feeding, after patients achieved $80 \%$ of the nutrition target calories $(25 \mathrm{kcal} / \mathrm{kg} / \mathrm{d})$ through continuous feeding, then the feeding/fasting time were reasonably determined, according to the circadian rhythm of the human body and the physiological movement of digestive organ in traditional Chinese medicine theory. In traditional Chinese medicine theory, each human organ works regularly in different times of one day, and the best feeding times were 7-9 o 'clock, 11-13 o 'clock and 17-19 o 'clock. So this sequential feeding consists of initial continuous feeding and subsequent intermittent feeding. We think this feeding method is more consistent with the law of human physiological activities and more suitable for critically ill patients.

Except for hypoglycemia ${ }^{[10]}$, high glucose value and high glycemic variability were also independent conditions associated with increased risk of mortality in critical illness ${ }^{[11,12]}$. The primary objective of this study was to determine if there is a difference in blood glucose value among critically ill patients receiving sequential feeding versus continuous feeding. Secondary objectives included glycemic variability and feeding intolerance. We hypothesized that the average of glucose in sequential feeding was no inferior to continuous feeding.

\section{Materials And Methods}

\section{Study design and ethical consideration}

We performed a prospective, single-blinding and non-inferiority Randomized Controlled Trial involving ICU patients in our hospital. The trial was approved by the Ethics Committee of the Affiliated Hospital of Qingdao University. This study is a part of a randomized controlled trial (clinicaltrials.gov, NCT03439618). All of the patients or their legally authorized representatives provided written informed consent.

\section{Patients}


This study was conducted on patients who had admitted to ICU from May to December 2018. Patients fed through gastric tubes were eligible for inclusion.

The exclusion criteria were:

1. Patients with the ability to eat orally;

2. Patients with diabetes, gastrointestinal disease or surgery;

3. Patients who are unable to tolerate enteral feeding or enteral feeding fail to meet the target dosage of $80 \%$.

4. The estimated feeding time is less than 7 days.

\section{Intervention}

Nutrition assessment qualification at initiation of enteral feeding was performed. The first assessment was generally completed in the first 24 hours, and a formal reassessment was performed every day while the subjects were in ICU. If it is estimated that 1 ) development of hemodynamic stability (mean arterial pressure $>65 \mathrm{mmHg}$, and gradually reduce the amount of vasoactive drugs); 2) Patients with NRS 2002 score $>3$, enteral nutrition treatment was performed.

At the beginning, all the patients were performed with continuous feeding. After achieved $80 \%$ of the nutrition target calories $(25 \mathrm{kcal} / \mathrm{kg} / \mathrm{d}$ ) through continuous feeding, the patients were randomly assigned into sequential feeding (SF) group or continuous feeding (CF) group with a random number table. Patients in SF group, the feeding/fasting time were reasonably determined, according to the circadian rhythm of the human body, and the physiological movement of digestive organs in traditional Chinese medicine theory. The total daily dosage of enteral nutrition was equally distributed to the three time periods of 7-9 o 'clock, 11-13 o 'clock and 17-19 o 'clock. Enteral nutritional suspension in each time period was pumped at a uniform rate within two hours by enteral feeding pump. While patients in CF group received continuous feeding with constant velocity by enteral feeding pump. Fingertip blood glucose values at 5 time points (6:00/11:00/15.00/21:00/1:00) were monitored and recorded in 7 consecutive days after randomization. Meanwhile, enteral feeding intolerance was recorded by a researcher-made checklist including the data on diarrhea, abdominal distension, constipation and gastric residual volume (GRV).

\section{Outcome Measures}

Baseline characteristics of the patients were recorded by a researcher-made checklist including age, sex, weight, major diagnosis, APEACH $\otimes$ scores and NRS 2002 at the beginning of the study. The primary outcome is the average blood glucose (GluAve) value among 7 days. Glycemic variability (GV) was the 
secondary outcome. GV was assessed by 3 indices: standard deviation of glucose (GluSD), coefficient of glucose variation (GluCV) and Glucose $\max _{\max }$-Glucose $\min _{\min }$ GluCV is GluSD divided by GluAve.

Enteral feeding intolerance was another secondary outcome. The researcher-made checklist was used for evaluating enteral feeding intolerance, including the data on diarrhea, abdominal distension, constipation, and gastric residual volume (GRV). In one of the following cases, enteral feeding intolerance was confirmed: (1) Diarrhea: the patient having three or more loose or continued to overflow loose stools within 24 hours ${ }^{[13,14]}$. (2) Abdominal distension: obvious abdominal distension or intra-abdominal pressure $\geq 12 \mathrm{mmhg}{ }^{[15]}$. (3) Constipation: fewer than 3 bowel movements per week, or exertion in defecation and hard stools ${ }^{[16]}$. (4) Gastric residual volume: GRV $\geq 200 \mathrm{ml}$ by ultrasonic monitoring, was the sign of intolerance ${ }^{[17]}$. In SF group, GRV was assessed after stopping the feeding pump for 2 hours (11:00/15:00/21:00). And in CF group, GRV was also assessed at this 3 points (11:00/15:00/21:00). Duration of mechanical ventilation, lengths of stay in the ICU, and ICU mortality rate were also recorded.

\section{Statistical Analysis}

Non-inferiority testing was adopted in this study. In continuous feeding, the blood glucose value was approximately $9 \mathrm{mmol} / \mathrm{L}$, Standard Deviation was about 3 . We speculated the blood glucose value was also $9 \mathrm{mmol} / \mathrm{L}$. Then a was set at $0.05,1-\beta$ was set at 0.8 , the non-inferiority value was set 2 , and the calculated sample size was 28 in each group. Another 6 patients were needed to correct an expected $10 \%$ drop out.

All statistical analyses were performed by SPSS software version 22.0 (SPSS, Inc. Chicago,IL, USA). Date were expressed as mean \pm standard deviations for quantitative variables and proportions for categorical and binary variables. Student $t$ test was used for statistical comparison between groups for continuous variables. The $\chi^{2}$ test or Fisher test was used for comparison between groups for count variables. $P$ values $₫ 0.05$ was considered as statistically significant.

\section{Results}

A total of 903 patients assessed for eligibility in the study from March to December 2018, and 62 patients were finally enrolled (Figure 1). 32 patients were randomized for SF group and 30 patients were randomized for CF group.

\section{General and clinical characteristics}

Baseline characteristics of the patients in the two groups were presented in Table 1. There were no

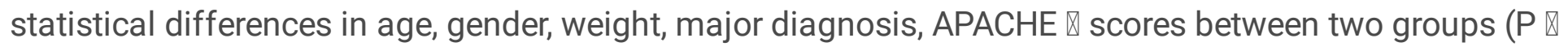
0.05). 


\section{Primary and secondary outcomes}

The average of glucose in sequential feeding was no inferior to continuous feeding [8.8(7.3-10.3) $\mathrm{mmol} / \mathrm{L}$ vs. $10.7(9.1-12.1) \mathrm{mmol} / \mathrm{L}, \mathrm{P}$ for non-inferiority $=0.038]$. There were differences in coefficient of glucose variation between two groups [24.5(22.2-27.6) \% vs. 18.9(13.7-25.3) \%, P=0.013]. But there was no significant differences in glucose standard deviations and $\mathrm{Glu}_{\max }-\mathrm{Glu}_{\min }$ between two groups [2.2(1.6-3.1) vs. 2.3(0.9-2.6), $P=0.489 ; 7.5(6.1-10.9)$ vs. 8.7(3.9-10.4), $P=1.000]$. The mechanical ventilation days in SF group were less than CF group [10(4-23) vs. 17(10-28), $P=0.030]$. Moreover, no significant differences in the incidence of feeding intolerance during the 7 days, length of stay in ICU and mortality in ICU were found $(P>0.05)$.

At $7^{\text {th }}$ day, incidence of insulin therapy was no significant difference in both groups $(8 / 32 \mathrm{vs} .10 / 30, P=$ 0.470). At 6:00,11:00, 15:00 and 1:00 time points, the average glucose value in SF group was lower than CF group (P区0.05). Moreover, hypoglycemia (Glucose $\varangle 3.9 \mathrm{mmol} / \mathrm{L}$ ) was not found in both groups. But the hyperglycemia (Glucose $₫ 11.1 \mathrm{mmol} / \mathrm{L}$ ) in CF group was more often than SF group (36.1\% vs. 23.0\%, P区 0.01).

\section{Discussion}

In the present study, the average of glucose in sequential feeding was no inferior to continuous feeding. In addition, hyperglycemia in CF group was more often than SF group. The mechanical ventilation days in SF groups were less than that in CF group. There was no increase in feeding intolerance in sequential feeding. However, glycemic variability in sequential feeding group may be higher than continuous feeding group.

A consensus has been reached on the importance of nutritional support for critically ill patients. However, the choice of enteral nutrition feeding method for ICU patients is still controversial. Continuous feeding was thought to be better tolerated by critically ill patients with gastrointestinal dysfunction, easy insulin control and limited absorptive gut surface area ${ }^{[5]}$. While, when patients achieved $80 \%$ of the nutrition target volume, gastrointestinal function is almost restored. In this time point, it comes a question that whether continuous feeding is necessary for patients all the time or not.

Critically ill patients usually develop high glucose value, which was associated with adverse outcome ${ }^{[11]}$. The study shows that average of glucose (GluAve) of SF group, was not higher than CF group. And blood glucose level in subjects of SF group at 6:00,11:00, 15:00 and 1:00 was lower than CF group. In addition, the incidence of hyperglycemia in SF group was less than CF group. And hypoglycemia did not occur in SF group.

Avoidance of glucose variability causing negative outcomes, was a priority for patients in ICU ${ }^{[18]}$. It is the belief of many clinicians that continuous, rather than intermittent feeding helps to reduce this variability. However, in a recent study of 50 critically ill patients comparing bolus feeding given via a percutaneous 
endoscopic gastrostomy tube with continuous feeding, no difference in glucose variability or insulin utilization was found ${ }^{[5]}$. Our study also suggested there were no significant differences in glucose standard deviations and Glucose $\max$-Glucose $_{\min }$ between two groups. But we also found coefficient of glucose variation in SF group was higher than CF group. Actually previous studies have shown that glucose variability affects the prognosis of critically ill patients ${ }^{[18]}$, but when patients are able to tolerate $80 \%$ enteral nutrition, it often indicates that their condition tends to be stable, and at this time, the reasonable fluctuation of blood glucose may be beneficial to patients since it was proved that reasonable glucose variability causes infusion fluctuation, which has beneficial to protein synthesis ${ }^{[19]}$. Besides, the previous studies about glucose variability were all bout continuous feeding, the influence of glucose variability in continuous feeding and sequential feeding may be different. In this study, the difference of mortality was not found. And sequential feeding had the advantage of reducing the mechanical ventilation days. Advanced studies are needed to confirm the effect of glucose variability in sequential feeding.

Feeding intolerance is very common in patients in ICU, especially in terms of delayed gastric emptying. It has been shown that both the rate and extent of nutrient absorption is impaired, even when postpyloric feeding ${ }^{[20]}$. A study has yet to be measured in critically ill patients, intermittent feeding may increase gut motility by increasing superior mesenteric artery blood flow, and enhancing cholecystokinin and peptide YY concentrations ${ }^{[21]}$. Similar to other study ${ }^{[3-4]}$, our study also suggest that no significant differences in the incidence of feeding intolerance.

Appropriate nutrition, where energy intake is aligned with energy expenditure and clear feeding/fasting cycles are synchronised with clock-regulated metabolic changes, helps maintain robust behavioural and physiological circadian rhythms and health ${ }^{[22]}$. Circadian misalignment is detrimental to metabolism [23]. Patients in ICU, as a special population, are characterized by severity and complexity of the disease. Their circadian rhythm is often changed by severe physical and mental trauma, stress and sleep disorders. Moreover, continuous nutritional delivery alters circadian rhythms of intestinal hormones, and postprandial rhythms of ghrelin and insulin release in response to nutrition ${ }^{[24]}$. These patterns of altered physiology might be avoided by preventing reprogramming of the circadian rhythm using an intermittent feeding regimen, but studies are needed to confirm this hypothesis. The selection of time points in sequential feeding is based on the theory of human body clock in traditional Chinese medicine, and we thought this sequential feeding was also benefit for ICU patients. The potential benefits of circadian rhythm in sequential feeding is one of theoretical basis for our ongoing study of RCT study about protein synthesis (NCT03439618).

Continuous feeding may have the following advantages [20]: better GRV management in some populations, better blood sugar control in early stages of critical illness, less labour intensive for nursing staff and wider choice of enteral feeds. On the other hand, intermittent feeding has the following advantages ${ }^{[20]}$ : enhancing muscle protein synthesis, ability to "catch-up" on missed feeds, reduction in diarrhoea, allows freedom during rehabilitation and other procedures. Sequential feeding in this study 
may integrate the advantages of continuous feeding and intermittent feeding. Continuous feeding is used at the initial stage when gastrointestinal function is impaired, and intermittent feeding, which in line the circadian rhythm of the human body, is used after almost gastrointestinal function recovery. This study demonstrated that average of glucose and EF intolerance of patients in ICU was similar in both sequential feeding and continuous feeding. It suggested that sequential feeding maybe as safe as continuous feeding for patients in ICU.

There were some limits in this paper. First, although the value of GluAve in sequential feeding was lower than continuous feeding, it cannot completely demonstrate that control blood glucose in sequential feeding is better than continuous feeding because it is a non-inferiority study. Second, lots of patients were excluded from these excluded factors, such as diabetes and gastrointestinal diseases, and further studies are needed to determine whether the results of this study are suitable for such patients. Third, the observation period is 7 days, and whether the conclusion can be reached in a shorter period, such as 3 days, requires further study.

\section{Conclusion}

In this non-inferiority study खblood glucose average value of critically ill patients with sequential feeding was not higher than continuous feeding. Compared to continuous feeding, hyperglycemia was less in sequential feeding. And the mechanical ventilation days were less in sequential feeding. So sequential feeding may be as safe as continuous feeding for critically ill patients.

\section{Declarations}

\section{Conflicts of Interest and Source of Funding:}

The authors declare that they have no competing interests, and this study was supported by Projects of medical and health technology development program in Shandong province (N0.2018WSB26018)

\section{Conflict of interest}

The authors declare no conflict of interest related to this work.

\section{Authors' contributions}

Xu-feng Pang and Bo Yao designed the study and modified the manuscript. Chang-jie Ren performed the study and drafted the manuscript. All authors read and approved the final manuscript.

\section{Acknowledgements}




\section{References}

[1] Di Girolamo FG, Situlin R, Fiotti N, Biolo G. Intermittent vs. continuous enteral feeding to prevent catabolism in acutely ill adult and pediatric patients. Current Opinion in Clinical Nutrition Metabol Care. 2017,20(5):390-395.

[2] Sunderram J, Sofou S, Kamisoglu K, Karantza V, Androulakis IP. Time-restricted feeding and the realignment of biological rhythms: translational opportunities and challenges. J Transl Med. 2014,12:79.

[3] Bowling TE, Cliff B, Wright JW, Blackshaw PE, Perkins AC, Lobo DN. The effects of bolus and continuous nasogastric feeding on gastro-oesophageal reflux and gastric emptying in healthy volunteers: a randomised three-way crossover pilot study. Clinical nutrition. 2008, 27(4):608-613.

[4] Tavares de Araujo VM, Gomes PC, Caporossi C. Enteral nutrition in critical patients; should the administration be continuous or intermittent? Nutr Hosp. 2014, 29(3):563-7.

[5] Evans DC, Forbes R, Jones C, Cotterman R, Njoku C, Thongrong C, Tulman D, Bergese SD, Thomas S, Papadimos TJ, Stawicki SP. Continuous versus bolus tube feeds: Does the modality affect glycemic variability, tube feeding volume, caloric intake, or insulin utilization?.International Journal of Critical Illness \& Injury Science, 2016, 6(1):9-15.

[6] Goo RH, Moore JG, Green berg E, Alazraki N P. Circadian variation in gastric emptying of meals in humans. Gastroenterology. 1987,93:515-518.

[7] Rao SS, Sadeghi P, Beaty J, Kavlock R, Ackerson K. Ambulatory 24-h colonic manometry in healthy humans. Am J Physiol Gastrointest Liver Physiol. 2001, 280(4):G629-39.

[8] Han S, Zhang R, Jain R, Shi H, Zhang L, Zhou G, Sanqwunq P, Tuqal D, Atkins GB, Prosdocimo DA, et al. Circadian control of bile acid synthesis by a klf15-fgf15 axis. Nat Commun. 2015,6:7231.

[9] Potter GD, Cade JE, Grant PJ, Hardie LJ. Nutrition and the circadian system. British Journal of Nutrition. 2016,116(3):434-442.

[10] Saliba L, Cook CH, Dungan KM, Porter K, Murphy CV. Medication-induced and spontaneous hypoglycemia carry the same risk for hospital mortality in critically ill patients. Journal of Critical Care. 2016,36:13-17.

[11] Bagshaw SM, Egi M, George C, Bellomo R. Early blood glucose control and mortality in critically ill patients in Australia. Critical Care Medicine. 2009,37(2):463-70.

[12] Todi S, Bhattacharya M. Glycemic variability and outcome in critically ill. Indian J Crit Care Med. 2014,18(5):285-90 
[13]Britoashurst D, Ione I. Diarrhea in Critically III Patients: The Role of Enteral Feeding. Jpen Journal of Parenteral \& Enteral Nutrition, 2016, 40(7):913-923.

[14] Reintam Blaser A, Malbrain ML, Starkopf J, Fruhwald S, Jakob SM, De Waele J, Braun JP, Poeze M, Spies C. Gastrointestinal function in intensive care patients: terminology, definitions and management. Recommendations of the ESICM Working Group on Abdominal Problems. Intensive Care Medicine. 2012, 38(3):384-394.

[15] Reintam Blaser A, Starkopf J, Malbrain ML. Abdominal signs and symptoms in intensive care patients. Anaesthesiology Intensive Therapy. 2015, 47(4):379-387.

[16] Locke GR, Pemberton JH, Phillips SF. American Gastroenterological Association Medical Position Statement: guidelines on constipation. Gastroenterology. 2000, 119(6):1761-1766.

[17] Elke G, Felbinger TW, Heyland DK. Gastric Residual Volume in Critically III Patients: A Dead Marker or Still Alive? Nutrition in Clinical Practice. 2015, 30(1):59-71.

[18] Hermanides J, Vriesendorp TM, Bosman RJ, Zandstra DF, Hoekstra JB, Devries JH. Glucose variability is associated with intensive care unit mortality. Critical Care Medicine. 2010,38(3):838-842.

[19] Gazzaneo MC, Suryawan A, Orellana RA, Torrazza RM, El-Kadi SW, Wilson FA, Kimball SR, Srivastava $\mathrm{N}$, Nquyen HV, Fiorotto ML, Davis TA. Intermittent bolus feeding has a greater stimulatory effect on protein synthesis in skeletal muscle than continuous feeding in neonatal pigs. Journal of Nutrition. 2011, 141:2152-2158.

[20] Bear DE, Hart N, Puthucheary Z. Continuous or intermittent feeding: pros and cons. Current Opinion in Critical Care. 2018, 24(4):256-261.

[21] Chowdhury A H, Murray K, Hoad C L,Costiqan C, Macdonald IA, Bowling TE, Lobo DN. Effects of Bolus and Continuous Nasogastric Feeding on Gastric Emptying, Small Bowel Water Content, Superior Mesenteric Artery Blood Flow, and Plasma Hormone Concentrations in Healthy Adults. Annals of Surgery. 2016, 263(3):450-457.

[22] Chaix A, Zarrinpar A, Miu P, Panda s. Time-Restricted Feeding Is a Preventative and Therapeutic Intervention against Diverse Nutritional Challenges. Cell Metabolism. 2014, 20(6):991-1005.

[23] Johnston JD, Ordovas JM, Scheer FA, Turek FW. Circadian rhythms, metabolism, and chrononutrition in rodents and humans. Advances in Nutrition: An International Review Journal, 2016, 7(2):399-406.

[24] Chowdhury AH, Murray K, Hoad CL, Costiqan C, Marciani L, Macdonald IA, Bowling TE, Lobo DN. Effects of bolus and continuous nasogastric feeding on gastric emptying, small bowel water content, superior mesenteric artery blood flow, and plasma hormone concentrations in healthy adults: a randomized crossover study. Annals of Surgery. 2016, 263(3):450-457. 


\section{Tables}

Table 1 Comparison of general and clinical characteristics of the patients between the two groups

\begin{tabular}{rccc}
\hline Variables & SF group $(\mathrm{n}=32)$ & CF group $(\mathrm{n}=30)$ & P value \\
\hline Age (years) & $66 \square 54-72 \square$ & $55 \square 48-67 \square$ & 0.062 \\
gender (male/female) & $17 / 15$ & $19 / 11$ & 0.416 \\
Weight (Kg) & $65 \square 60-78 \square$ & $65 \square 60-87 \square$ & 0.613 \\
APACHE II scores & $19 \square 15-23 \square$ & $16 \square 10-19 \square$ & 0.059 \\
Major diagnosis & & & \\
Pneumonia & $13 / 32$ & $12 / 30$ & 0.960 \\
Brain diseases & $10 / 32$ & $9 / 30$ & 0.915 \\
& & & \\
( stroke, hemorrhage or infection) & & $5 / 30$ & 0.249 \\
Trauma & $2 / 32$ & $2 / 30$ & 0.607 \\
Cardiac arrest & $1 / 32$ & $1 / 30$ & 1.000 \\
Hepatobiliary infection & $2 / 32$ & $1 / 30$ & 0.355 \\
Others & $4 / 32$ & &
\end{tabular}

Table 2 Comparison of outcomes between the two groups

\begin{tabular}{cccc}
\hline Variables & SF group $(\mathrm{n}=32)$ & CF group $(\mathrm{n}=30)$ & P value \\
\hline GluAve $(\mathrm{mmol} / \mathrm{L})$ & $8.8(7.3-10.3)$ & $10.7(9.1-12.1)$ & 0.038 \\
GluSD (mmol/L) & $2.2(1.6-3.1)$ & $2.3(0.9-2.6)$ & 0.489 \\
GluCV (\%) & $24.5(22.2-27.6)$ & $18.9(13.7-25.3)$ & 0.013 \\
Glu $_{\text {max }}-$ Glu $_{\min }(\mathrm{mmol} / \mathrm{L})$ & $7.5(6.1-10.9)$ & $8.7(3.9-10.4)$ & 1.000 \\
Incidence of feeding intolerance & $4 / 32$ & $2 / 30$ & 0.672 \\
Mechanical ventilation days & $10(4-23)$ & $17(10-28)$ & 0.030 \\
LOS in ICU & $22(12-27)$ & $25(12-31)$ & 0.433 \\
Mortality in ICU & $2 / 32$ & $2 / 30$ & 1.000 \\
\hline
\end{tabular}

Table 3 Comparison of average blood glucose between the two groups in five time points

\begin{tabular}{cccc}
\hline Variables & SF group (n=32) & CF group $(\mathrm{n}=30)$ & P value \\
\hline 6:00 GluAve(mmol/L) & $6.7(6.0-7.4)$ & $8.4(6.7-10.2)$ & $\square 0.001$ \\
11:00 GluAve(mmol/L) & $8.8(6.9-12.1)$ & $10.8(8.2-12.4)$ & 0.001 \\
15:00 GluAve(mmol/L) & $9.6(7.1-11.9)$ & $10.808 .1-13.0 \square$ & 0.007 \\
21:00 GluAve(mmol/L) & $10.2(7.8-12.2)$ & $9.9(8.0-12.0)$ & 0.969 \\
1:00 GluAve(mmol/L) & $7.4(6.3-9.3)$ & $10.0(7.9-11.7)$ & $\square 0.001$ \\
\hline
\end{tabular}

\section{Figures}




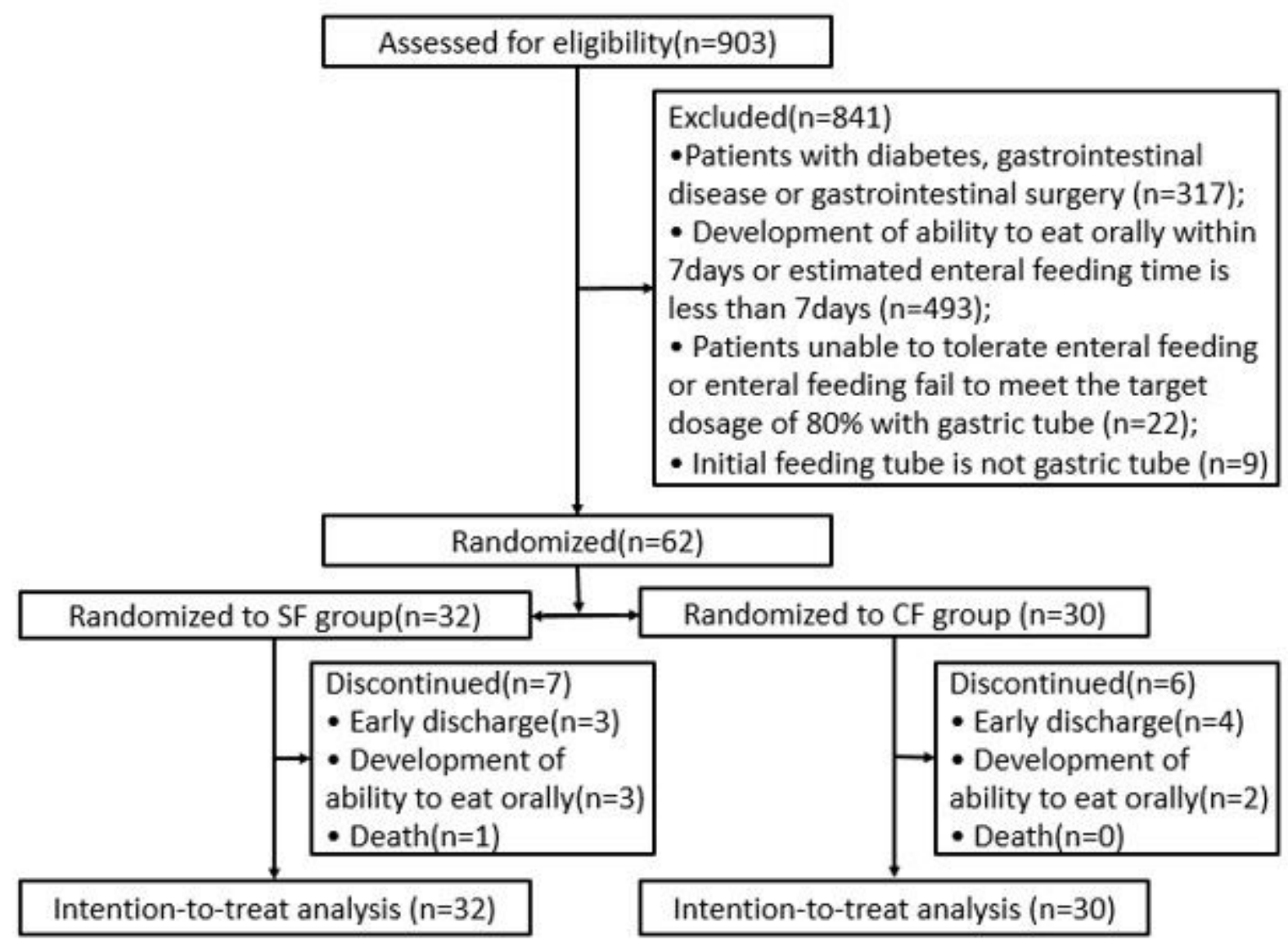

\section{Figure 1}

Flow diagram of subjects 